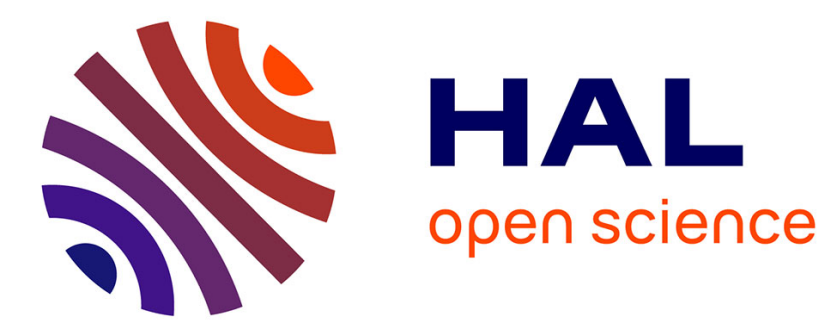

\title{
Risques psychosociaux et santé au travail
}

\author{
Valérie Saint-Dizier de Almeida, Gérard Vallery
}

\section{To cite this version:}

Valérie Saint-Dizier de Almeida, Gérard Vallery. Risques psychosociaux et santé au travail. Psychologie du travail et des organisations, 2017, 23 (4), pp.273-277. 10.1016/j.pto.2017.08.006 . hal02042377

\section{HAL Id: hal-02042377 \\ https://hal.science/hal-02042377}

Submitted on 18 Oct 2019

HAL is a multi-disciplinary open access archive for the deposit and dissemination of scientific research documents, whether they are published or not. The documents may come from teaching and research institutions in France or abroad, or from public or private research centers.
L'archive ouverte pluridisciplinaire HAL, est destinée au dépôt et à la diffusion de documents scientifiques de niveau recherche, publiés ou non, émanant des établissements d'enseignement et de recherche français ou étrangers, des laboratoires publics ou privés. 


\section{Justification du Numéro spécial PTO « Risques psychosociaux et santé au travail » Valérie Saint-Dizier de Almeida (1) \& Gérard Valléry (2) \\ (1) Université de Lorraine, CLSH, BP 3397, 54015 Nancy Cedex, France, valerie.saint-dizier@univ-lorraine.fr \\ (2) Université de Picardie, CRP, Chemin du Thil, 80025 Amiens Cedex 1, France gerard.vallery@wanadoo.fr}

L'évaluation et la prévention des risques psychosociaux (RPS) ont pour finalité d'assurer une meilleure protection des salariés et de concourir à leur bien-être tout en s'inscrivant dans une recherche d'efficacité globale des organisations. Les RPS peuvent être générés par différentes causes issues notamment des contraintes du travail, de l'organisation, d'un manque de reconnaissance, des valeurs, des relations conflictuelles entre collègues ou avec la hiérarchie... Le champ des relations de service est encore davantage impacté dans le sens où peuvent apparaître des risques provenant des interactions entre agents et usagers/clients/bénéficiaires. Les RPS peuvent avoir des retentissements négatifs sur plusieurs plans (sur les personnes, l'organisation et le contenu du travail ou la qualité du service rendu). Les articles proposés s'inscrivent dans ce champ.

Généralement, l'identification et la prévention des risques reposent sur deux phases successives. La première vise l'identification de situations à risque et leur classification relativement à leur dangerosité et à leur fréquence (phase de diagnostic). La seconde vise à développer des préconisations sous forme d'aménagements/solutions qui permettront de réduire/faire disparaître les facteurs de risques ciblés et à les mettre en œuvre (phase du plan d'actions).

La problématique des RPS est relativement récente. Depuis une dizaine d'année, des chercheurs en psychologie du travail développent des programmes à des fins de recherche ou d'interventions. Dans ce cadre, des investigations scientifiques ont conduit au développement de méthodes quantitatives et qualitatives ; certaines se centrent sur des diagnostics, d'autres sur des plans d'actions et d'autres encore combinent les deux dans le processus d'intervention, selon la nature de la demande et la temporalité de la démarche engagée. La diversité des études dépend également du champ d'expertise des chercheurs et/ou du contexte organisationnel dans lequel elles sont conduites. Les sept articles qui composent ce numéro représentent une partie de cette diversité. Le numéro a pour visée d'éclairer les chercheurs et praticiens sur les apports et limites de méthodologies actuellement disponibles dans le champ des RPS. Ils restituent différents protocoles de recherche ou d'intervention exploitant des méthodes qui sont discutées au vu des contextes spécifiques et des résultats auxquels elles aboutissent.

Le premier article produit par Sandrine Ponnelle et Xenophon Vaxevenoglou dresse un état de l'art sur la question des RPS et de la santé au travail. L'enjeu est d'expliquer comment, dans le contexte français, un consensus méthodologique articulant législation européenne et accords nationaux, théories scientifiques et instruments d'évaluation, et prise en compte du travail réel s'est progressivement établi. Au terme de cette étude, les auteurs montrent qu'une tendance est, dans le cadre de la prévention primaire, de fournir aux salariés les ressources pour qu'eux-mêmes via une activité réflexive, agissent sur leur environnement de travail pour contrer l'émergence de facteurs à risques.

Les articles qui suivent restituent des études et mettent le focus sur la dimension méthodologique des processus d'intervention. Les différentes méthodologies mobilisées sont mises en œuvre dans différents secteurs d'activité (soins, social, collectivité locale,...) et visent l'instruction des risques et/ou leur prévention. Certaines sont quantitatives, d'autres qualitatives ou articulent les deux.

Plus précisément, Jérémy Lemoine, Frédéric Deschamps et Christine Roland-Lévy présentent un questionnaire destiné aux professionnels de la santé qu'ils ont élaboré sur la base des six dimensions identifiées par le Collège d'expertise. 
La comparaison de leur questionnaire au questionnaire spécifiquement développé pour les personnels de santé (ORSASA) révèle que celui conçu par les auteurs, permet de prédire davantage le stress et le burnout qu'une mesure des contraintes psychosociales et organisationnelles, comme celle d'ORSASA. Sur la base des résultats qu'ils ont obtenus, les auteurs envisagent de peaufiner leur outil en créant des items qui permettrait de distinguer les dimensions conflits de valeur et intensité du travail et temps de travail, d'identifier une dimension autonomie en plus de la dimension compétence, de différencier les relations hiérarchiques des relations avec les collègues.

L'article de Alison Caillé et Christine Jeoffrion montre l'apport d'une méthodologie mixte et participative reposant sur des observations participantes, des entretiens individuels et collectifs, et des associations libres. La méthodologie est exploitée pour la prévention des RPS au sein d'une collectivité territoriale. Si habituellement l'approche participative est mise en place une fois le diagnostic établi en vue de développer collectivement des modes d'action visant à atténuer/supprimer des facteurs de risque ; ici elle est mise en place en amont du processus d'intervention. Cette option s'est traduite par la constitution d'un comité de pilotage représentatif des principaux acteurs en amont du processus d'intervention et d'un plan de communication efficace visant à informer les salariés des résultats produits à chacune des étapes. La mise en place de cette option a permis de recueillir des données représentatives de la population étudiée avec un taux de participation rarement atteint dans ce genre d'études ( $91 \%$ de taux de participation au questionnaire) et montre l'importance d'impliquer les salariés dès le début de l'intervention.

Corinne Ribert-Van de Weerdt présente une recherche-action dans le secteur de l'aide à domicile et montre comment des mesures préventives ont été développées. Cette recherche met le focus sur la dimension émotionnelle des activités de travail. Dans un objectif de prévention, vis-à-vis des situations à risque de forte charge émotionnelle, sont examinées et étudiées d'une part les exigences du travail, essentiellement de nature émotionnelle, et leurs effets sur l'activité, et d'autre part les régulations individuelles et collectives des émotions en situation réelle, afin que des transformations puissent s'opérer dans une perspective de prévention. Dans cette optique, l'auteur développe une méthodologie spécifique exploitant l'auto-confrontation collective couplée à des entretiens et observations. De cette étude, il ressort que 1) les exigences du travail pour les salariés à domicile sont constituées d'un ensemble cumulé de déterminants techniques et organisationnels, dont la part émotionnelle est importante, 2) les exigences émotionnelles créent une charge émotionnelle, qui, si elle n'est pas régulée de façon fonctionnelle dans des conditions optimales, peuvent devenir trop fortes et engendrer à terme des problèmes de santé, 3) la mise à disposition et le renforcement des ressources individuelles, collectives et organisationnelles permettent aux individus et collectifs de réguler la charge émotionnelle pour la maintenir à un niveau acceptable, et d'agir plus directement sur le travail et ses déterminants.

Gérard Valléry et Sylvain Leduc dans le cadre des CAF, montrent comment l'étude de violences verbales à partir d'entretiens et de saisies d'interactions à l'accueil et au guichet permet l'identification de facteurs de risques et le repérage de stratégies de régulation de l'agent (et de la relation à l'usager) qui impactent l'organisation et la qualité du service.

Nathalie Erudel et Valérie Saint-Dizier de Almeida mettent le focus sur les techniques d'animation des réunions qui visent le développement de plans d'action dans une optique de régulation de risques identifiés en amont. L'intervention s'est déroulée en milieu hospitalier. L'identification des risques repose sur l'utilisation du questionnaire SATIN ; leur régulation, sur la mise en place de réunions impliquant un collectif de 8 à 10 agents en moyenne qui s'est réuni à trois reprises. Chaque réunion exploite une forme d'animation spécifique : animation avec relances focalisées sur les causes, animation avec relances sur les solutions et animation enrichie de l'expertise du psychologue. L'enjeu est de caractériser ces méthodes d'animation et d'étudier leur impact sur l'activité collective. L'étude procède en des analyses des productions langagières produites au cours des trois réunions. Les résultats montrent notamment que 1) le style du discours est argumentatif quelle que soit la forme 
d'animation, 2) la nature des relances et l'enjeu de la réunion ont une incidence sur la structure fonctionnelle des réunions et 3) les relances sur les solutions induisent l'évocation de causes factuelles alors que les relances sur les causes conduisent à identifier des causes plus générales.

Enfin, Ludovic Tendille et Valérie Saint-Dizier de Almeida, à travers leur article montrent que la phase d'identification des risques physiques, lorsqu'elle implique des collectifs d'opérateurs concernés, est propice à la mise en exergue de risques psycho-sociaux. Ils montrent à travers l'étude d'une séquence extraite de la transcription d'une réunion dans une entreprise de productions de mailings publicitaires comment la régulation des risques physiques peut nécessiter la régulation de risques psychosociaux. Leur étude débouche sur différents enseignements : 1) si le processus d'identification des risques (fondés sur l'emploi d'outils comme les questionnaires) induit une dichotomie entre risques physiques et risques psychosociaux, cette distinction doit disparaître lorsqu'il s'agit de développer des actions visant la réduction ou la suppression des risques, 2) l'importance de sensibiliser les animateurs de tels groupes à l'identification des RPS et les former à la production de relances visant à instruire davantage le versant socio-émotionnel des situations investies, 3) l'importance de créer des groupes pour le traitement des risques professionnels : ces groupes permettent d'investir collectivement chacune des situations à risques, d'identifier leurs causes et de pouvoir partager une compréhension des situations, nécessaires à des solutions tenables ; 4) l'importance de réfléchir en amont à la composition des groupes de réflexion, ce qui suppose une étude de l'organisation du travail, des relations inter et intra-équipe. 